\title{
FIRST RESULTS FROM VIRGO ON SOHO
}

C. FRÖHLICH ${ }^{1}$, B.N. ANDERSEN ${ }^{2}$, T. APPOURCHAUX ${ }^{3}$

G. BERTHOMIEU ${ }^{4}$, D.A. CROMMELYNCK ${ }^{5}$, V. DOMINGO ${ }^{3}$

A. FICHOT ${ }^{5}$, W. FINSTERLE ${ }^{1}$, M.F. GÓMEZ ${ }^{6}$, D. GOUGH ${ }^{7,8}$

A. JIMÉNEZ ${ }^{6}$, T. LEIFSEN ${ }^{9}$, M. LOMBAERTS ${ }^{5}$, J.M. PAP ${ }^{10,11}$

J. PROVOST ${ }^{4}$, T. ROCA CORTÉS ${ }^{6}$, J. ROMERO ${ }^{1}$

H-J. ROTH ${ }^{1}$, T. SEKII ${ }^{7}$, U. TELLJOHANN ${ }^{3}$

T. TOUTAIN ${ }^{4}$, C. WEHRLI ${ }^{1}$

1 Physikalisch-Meteorologisches Observatorium Davos

World Radiation Center, CH-7260 Davos Dorf

${ }^{2}$ Norwegian Space Centre, $\mathrm{N}-0309$ Oslo 3

${ }^{3}$ Space Science Department, ESTEC, NL-2200 AG Noordwijk

${ }^{4}$ Departement Cassini, URA CNRS 1362,

Observatoire de la Cote d'Azur, F-06304 Nice Cedex 4

${ }^{5}$ Institut Royal Météorologique de Belgique, B-1180 Bruxelles

${ }^{6}$ Instituto de Astrofisica de Canarias,

Universidad de La Laguna, E-38071 La Laguna, Tenerife

7 Institute of Astronomy, University of Cambridge, Cambridge CB3 OHA, UK

${ }^{8}$ Department of Applied Mathematics and Theoretical Physics, University of Cambridge, Cambridge CB3 9EW, UK

${ }^{9}$ Institute of Theoreretical Astrophysics, University of Oslo, N-0315 Oslo

10 Department of Physics and Astronomy, Division of Astronomy and Astrophysics, University of California, Los Angeles, CA90095-1562 U.S.A.

11 Jet Propulsion Laboratory, California Institute of Technology, Pasadena, CA 91109 U.S.A.

Abstract. First results from the 4-6 months observations of the VIRGO experiment (Variability of solar IRradiance and Gravity Oscillations) on the ESA/NASA Mission SOHO (Solar and Heliospheric Observatory) are reported. The time series are evaluated in terms of solar irradiance vari- 
ability, solar background noise characteristics and p-mode oscillations. The solar irradiance is modulated by the passage of active regions across the disk, but not all of the modulation is straightforwardly explained in terms of sunspot flux blocking and facular enhancement. The observed p-mode frequencies are more-or-less in agreement with earlier measurements, but it is interesting to note that systematic differences seem to exist between the observations in different colours. There is also evidence that magnetic activity plays a significant role in the dynamics of the oscillations beyond its modulation of the resonant frequencies. Moreover, by comparing the amplitudes of different components of p-mode multiplets, each of which are influenced differently by spatial inhomogeneity, we have found that activity enhances excitation.

\section{Introduction}

The aim of VIRGO is to determine the characteristics of pressure and internal gravity oscillations by observing irradiance and radiance variations, to measure the solar total and spectral irradiance and to quantify their variability over periods of days to the duration of the mission as described by Fröhlich et al.(1996). VIRGO contains two different active-cavity radiometers (DIARAD and PMO6-V) for monitoring the solar 'constant', two three-channel sunphotometers (SPM) for the measurement of the spectral irradiance at 402, 500 and $862 \mathrm{~nm}$ with a bandwidth of $5 \mathrm{~nm}$, and a lowresolution imager (Luminosity Oscillation Imager, LOI) with 12 'scientific' and 4 guiding pixels, for measuring the radiance distribution over the solar disk at $500 \mathrm{~nm}$.

SOHO was successfully launched on 2 December, 1995. VIRGO was switched on a few days later. The covers were kept closed with the lock released in order to outgas the experiment thoroughly during the following 7 weeks. Real observations with the radiometers started mid-January, and observations with the sunphotometers at the end of January 1996. At that time it was realized that the LOI cover could not be opened. A concerted effort, as described by Appourchaux et al.(1997a), was needed, and finally on 27 March the cover was successfully opened. The LOI has been operating continuously since the end of March 1996. The performance of all the instruments is very good.

The observational data products are time series of the total and spectral irradiance and the LOI radiances which are corrected for all a priori known effects such as temperature, pointing, instrument-sun distance and relative velocity (for level 1), and inferred effects such as degradation (for level-2 


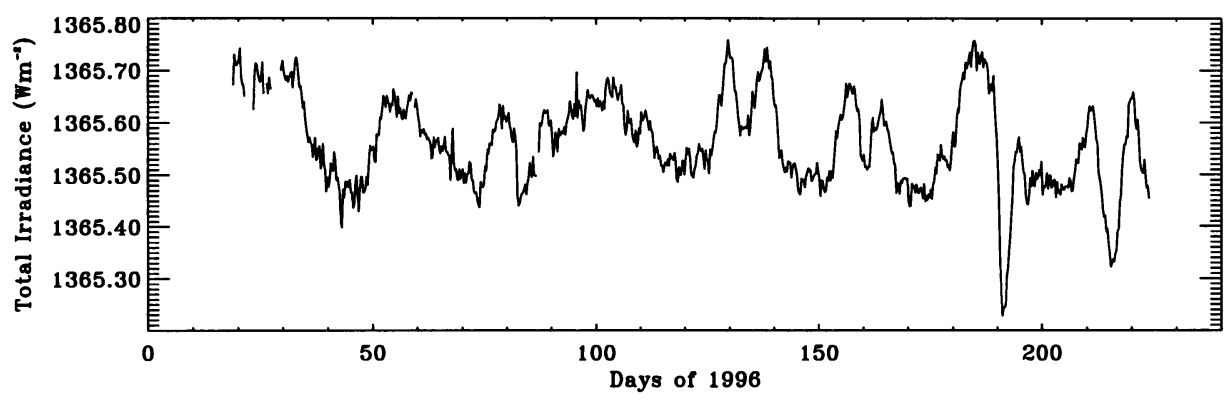

Figure 1. 6-hours averages of total solar irradiance in $\mathrm{Wm}^{-2}$ observed by VIRGO.

data products). The present scientific evaluation is carried out on preliminary level-2 data, which may change in the future owing to improvements in the production of level-2 data from level-1 data. Such datasets from the first 4-6 months of operation are the basis for the results we present here.

\section{Total and spectral solar irradiance and radiance measurements}

Observations of the total solar irradiance are a continuation of previous measurements from satellites, which have been performed by several experiments since the end of 1978 . The performance of the VIRGO radiometers and the procedures for the data evaluation are described by Fröhlich et al.(1997b). The radiometers are operated in active mode, and have a basic sampling cadence of 3 minutes for the DIARAD and of 1 minute for the PMO6-V. The continuously exposed left DIARAD channel shows no measurable degradation up to August 1996. This conclusion was obtained from several comparisons with the right DIARAD channel which is operated about every 60 th day for 30 minutes. In contrast, the operational PMO6-V radiometer degrades at a rate of $\approx 2.6 \mathrm{ppm} /$ day relative to DIARAD. The absolute irradiance values are based on the radiometric characterization of each radiometer obtained before launch. In space the two type of radiometers differ by about $1 \mathrm{Wm}^{-2}$, namely $0.07 \%$, well within the estimated absolute accuracy of each radiometer $(\approx 0.15 \%)$. The VIRGO solar irradiance values as shown in Fig. 1 are DIARAD values decreased by $0.5 \mathrm{Wm}^{-2}$ to account for the difference between both radiometers. The absolute values will be checked against measurements with similar radiometers on planned stratospheric balloon in spring 1997 and later with the experiment SOLCON onboard the NASA Hitchhiker program (Crommelynck et al.1996), and complemented by a detailed study of the time series of simultaneous measurements of VIRGO and ACRIM-II on UARS. 
The spectral irradiance data from the SPM are used to study the solar variability and solar oscillations. The performance of the SPM and the procedures for the data evaluation are described by Fröhlich et al.(1997b); here we summarize the most important characteristics. The sensitivity of the main instrument has slowly degraded with rates of about $-52 \mathrm{ppm} /$ day for the $862 \mathrm{~nm},-310 \mathrm{ppm} /$ day for the $500 \mathrm{~nm}$ and $-650 \mathrm{ppm} /$ day for the $402 \mathrm{~nm}$ channel. Compared to earlier space experiments with similar SPM these values are an order of magnitude smaller due to the strict cleanliness control of the SOHO mission. The spare instrument has been exposed only 4 times for 18-minute intervals, and shows no net trend; this is compatible with the observations of the total solar irradiance. The absolute values are about $2.9 \%$ below the ones of Neckel \& Labs (1984). While this is still within the combined errors of the NBS-1973 spectral irradiance scale of the lamps used and the reference spectrum of Neckel \& Labs (1984), the lower values of all channels hint at a systematic error during calibration. The absolute value will be checked during the stratospheric balloon flight planned for 1997 by comparing with a similar SPM calibrated before and after the flight, by means of the recently developed, very accurate radiometric method.

The variations seen in the time series of spectral and total irradiance are caused by a superposition of random and periodic phenomena. Variability caused by individual phenomena at the photosphere is modulated further by the solar rotation, leaving a very complicated mixture of temporal and spatial effects. Although the main objective of the LOI is the study of solar oscillations (Section 5), its spatial resolution allows us to deconvolve the effects of different spatial and temporal influence of photospheric variations on the solar irradiance. The performance of the LOI and the procedures for the data evaluation are described by Appourchaux et al.(1997a). The signals from the 16 pixels are corrected to fluxes measured at $1 \mathrm{AU}$ by taking into account the shape of the pixels, the limb darkening at $500 \mathrm{~nm}$, and the actual size of the solar image. In addition, for comparing the LOI integrated flux with the SPM, conversion factors are derived for the four type of pixels. The comparison of the LOI integrated flux with the SPM green channel shows that the LOI sensitivity decreases much faster with time. At the beginning of the exposure it amounted to about $1000 \mathrm{ppm} / \mathrm{day}$, decreasing to $500 \mathrm{ppm} /$ day after 100 days. The stronger degradation is probably due partly to the fact that the LOI entrance filter sees more solar wind particles and is partly a result of having different coatings on the front surfaces. 


\section{Solar noise}

The continuous measurements allow us to produce for the first time aliasfree power spectra of the variance of total and spectral irradiance over a wide range of frequencies corresponding to periods from 2 minutes to the duration of observation. This not only permits studies of the Sun with helioseismology, but also allows us to study the variability itself. In Fig. 2 the power density spectra of the SPM green channel is shown as an example. The most important features are:

- the p-mode signal stands above the solar background noise in all channels with a signal-to-noise at the peak of about 120:1 (in power);

- there is no visible excess in the solar noise in the p-mode region;

- in and above the p modes, the solar noise decreases with frequency as about $\nu^{-4}$; this value depends weakly on the wavelength of the observations, the blue and green channels being the steepest;

- from 10 to $100 \mu \mathrm{Hz}$ the solar noise decreases with frequency as $\nu^{-2}$;

- from 100 to $800 \mu \mathrm{Hz}$ the solar noise spectrum is quite flat; this was not expected, and it is a very encouraging finding because this is the range where $g$ modes are expected;

- a distinct hump in the power spectrum is seen at about $1.2 \mu \mathrm{Hz}$ in all spectra;

- fitting the spectra with function types proposed by Harvey (1985) gives time constants of the three regimes of the solar noise to be about 66,480 and 40000 seconds; this indicates that there are no structures visible with time constants in the mesogranulation regime; the shortest time constant could possibly be attributed to network bright points;

- at low frequencies peaks exist corresponding to periods of about 27, 9 and 7 days which are related to activity features crossing the visible disk.

Another new result is the frequency dependence of the ratio of the variance of the different spectral channels to that of total irradiance, which are quite complicated. In the frequency range of granulation and mesogranulation they are more or less constant; then they decrease towards lower frequencies with a broad minimum with about equal ratios for all colours at around $10 \mu \mathrm{Hz}$, and then at even lower frequencies there is a broad hump with the blue 10 times higher than the total and about 6 times higher than the red and green at periods of a few days.

\section{Influence of active regions on irradiance}

The time scales associated with the lifetime of magnetically active regions have revealed extremely interesting variations in total solar irradiance. 


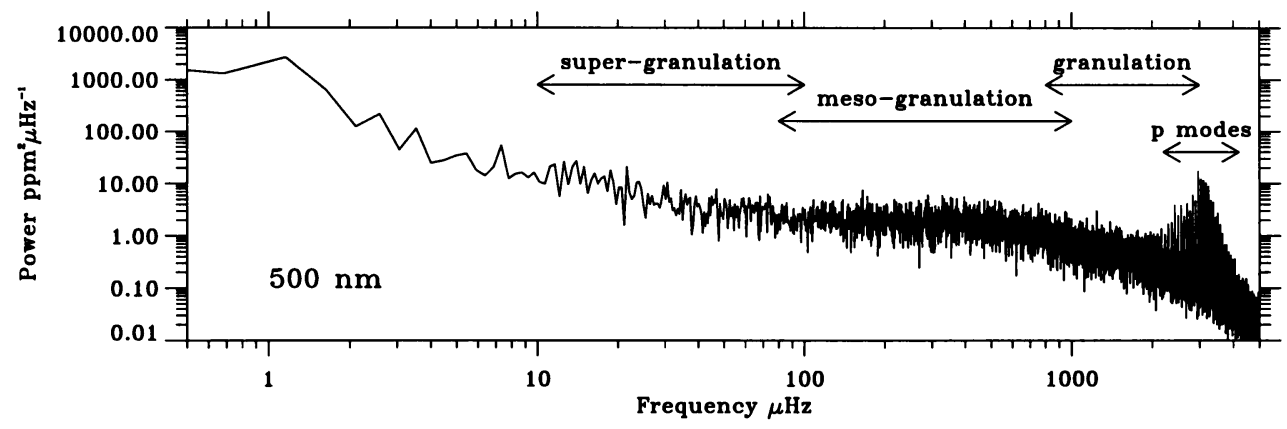

Figure 2. Power spectra of the green channel spectral irradiance time series for the period from January, 28 until August, 10 1996. The total, red and blue are very similar. The power ratios in the flat part of the spectrum $(50-200 \mu \mathrm{Hz})$ to the green are 0.47 , 0.64 and 1.93 for the total, red and blue.

Analyses based on former observations of total solar irradiance showed that the most striking events in the short-term irradiance changes are the sunspot-related temporary dips (Willson et al., 1981). The VIRGO experiment provides the first high-precision observations of solar spectral irradiance at 402,500 , and $862 \mathrm{~nm}$ in parallel with the total solar irradiance observations. These observations permit the study of simultaneous temporal changes in the near-UV, visible, and infrared ranges, and the determination of the spectral distribution of total solar irradiance variations, as is quite evident from Fig. 1. Here we discuss the occurrence of active region NOAA 7962 in early May 1996.

At the top of Fig. 3 we show magnetograms from Mt Wilson to illustrate the active region, and below we show time series of the total and spectral irradiance and of the four southern pixels of the LOI. The active region is causing significant changes in the spectral irradiance in the blue and green channels and the LOI, and much less in total irradiance and in the near IR. This particular active region appeared close to the limb in the southern part of the visible solar disk on 6 May (day 127) and contained a very small sunspot which vanished around 14 May. Very close to this region, a smaller active region without sunspots was also visible.

The LOI east pixel intensity value started to rise around 6 May with the appearance of the faculae in the active region rotating onto the east limb of the solar disk with a primary peak three days later. In parallel with the LOI east pixel intensity increase, both total solar and spectral irradiance in all the 3 wavelengths started to increase: by $\approx 300 \mathrm{ppm}$ for the blue, $\approx 200 \mathrm{ppm}$ for the green and $\approx 100 \mathrm{ppm}$ in both the red channel and the total irradiance. It is interesting to note that at the central meridian transit only the blue and green irradiances reached the quiet sun level, 

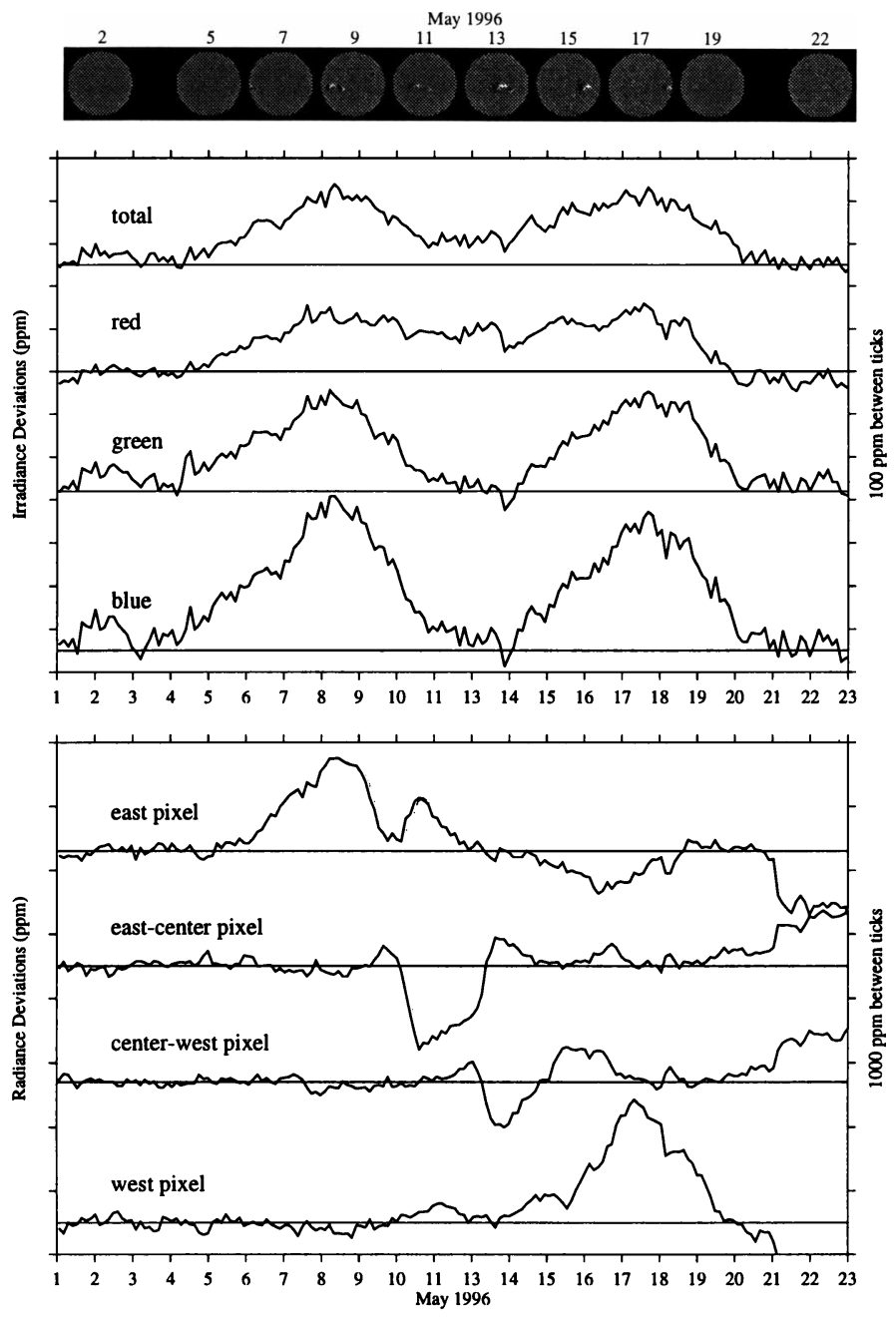

Figure 3. Passage of the active region NOAA 7962 in May 1996 shown by the Mt. Wilson magnetograms in the Fe I line (top panel, black and white correspond to \pm 5 Gauss), the time series of total and spectral irradiance (middle panel) and of the four LOI pixels south of the equator (bottom panel).

whereas the red channel and the total irradiance decreased by only about $40 \%$ and $60 \%$ of the maximal increase respectively. The photometric deficit of the spot group as determined by the San Fernando Observatory was around $50 \mathrm{ppm}$ (Solar-Geophysical Data, 1996) at the maximum sunspot influence (around May 12-13). Taking the wavelength dependence of the spot contrast into account one would expect 45,65 and $75 \mathrm{ppm}$ for red, green and blue channels respectively. This accounts for only about half of 
the observed decrease in total irradiance by the sunspot darkening. The other half must be due to the facular contribution which is smaller at disk center. Photometric observations of faculae show a limb brightening and the facular contrast at the disk centre is close to zero or is even negative. The behaviour close to the limb can account for the strong peak of the irradiance at central meridian distances of about $\pm 60^{\circ}$. The fact that only a partial decrease at the centre is observed indicates that the faculae are still seen in the spectral and total irradiance, or that the faculae extend over a much larger area than the magnetogram suggests. Moreover, the centreto-limb increase depends strongly on wavelength, and must be substantially smaller in the red and total irradiance than in the green and blue.

The LOI radiance values confirm this general behaviour. The east pixel shows mainly the increase due to faculae; on the crossover to the next pixel the radiance first increases due the faculae in front of the active region and then it suddenly decreases due to the dark spot. The effect of the $65 \mathrm{ppm}$ in the green sun-as-a-star signal is greater by a factor of about 12 , owing to the smaller area of the pixel, yielding a decrease down to about $-1100 \mathrm{ppm}$; then the spot slowly vanishes, indicated by the steady increase of the radiance. The change over to the next pixel shows again an increase and then the decrease due to the now smaller spot. At the central crossover there is still an increase, indicating that indeed the faculae are still bright close to the centre. This whole sequence shows very interesting details of how active regions influence the irradiance. Yet more detailed studies will definitively reveal clues for the understanding of irradiance and radiance variability.

\section{Determination of p-mode spectra}

The analysis is based on the power spectra calculated from the time series of the SPM channels and the 12 LOI pixels. The time series are detrended by piecewise fits of quadratic polynomials for the SPM and with a curve obtained by triangular smoothing with a full width of one day for the LOI. The power spectra are calculated from the Fourier transform of apodized time series by squaring the complex amplitude. As an example the p-mode spectrum from the SPM green channel is shown in Fig. 4. The amplitudes observed in the red, total and blue are $\approx 0.6,0.7$ and 1.4 times the green amplitude respectively.

\section{Frequencies of $\mathbf{p}$ modes}

From the SPM spectra the p-mode parameters are derived by fitting Lorentzians using a maximum-likelihood method as described in Toutain and Fröhlich (1992). The only difference is that the mode amplitudes in a given 


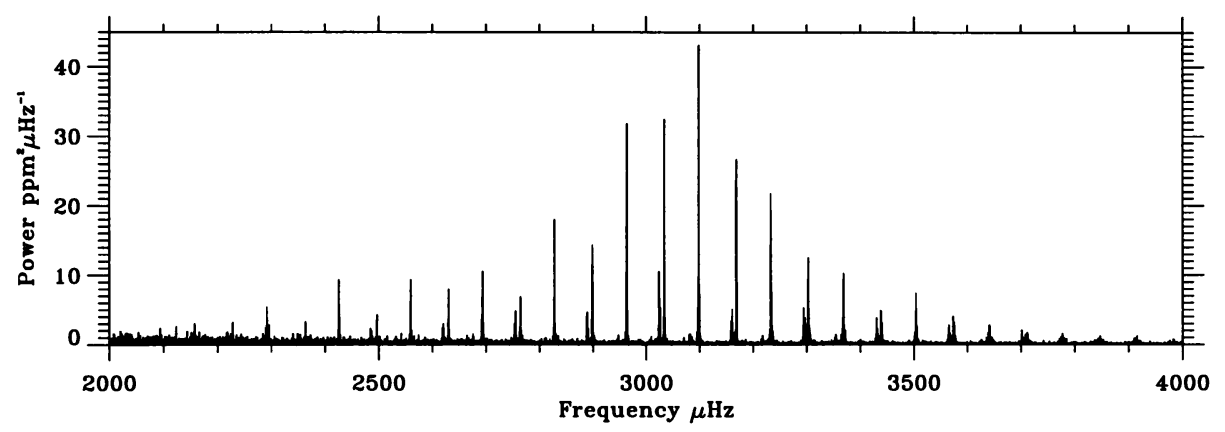

Figure 4. p-mode spectra from about 200 days of observation of the green irradiance. Note the high signal-to-noise ratio of about 120:1 (power).

$(l, m)$ multiplet are no longer constrained to be the same. For the LOI a given $(m, \nu)$ diagram is fitted simultaneously using a maximum-likelihood method as described in Appourchaux et al.(1995). Moreover, the crosstalk between the $2 l+1$ components of a multiplet as well as the aliases from higher-degree modes are appropriately taken into account (Appourchaux et al., 1997a).

The most important parameters of the p-mode characteristics are the frequencies. Five such sets are available in VIRGO. As the degeneracy splitting is not resolved in the low-degree modes determined from global observations of the sun the frequencies of the $l=1,2$ from the SPM or total spectra can be biased. The frequencies of the uninfluenced $l=0$ modes determined from the SPM and the total channels are in good agreement among themselves and with the ones determined from the LOI. In order to have an internally consistent data set we present as VIRGO first-result frequencies those determined from LOI. These frequencies are listed in Table 1. They can be compared with those determined by the IPHIR experiment (Toutain \& Fröhlich, 1992) during the second half of 1988. It is interesting to note that the frequencies of the $l=1$ modes are systematically higher, with a slight increase towards higher frequencies, which could well be due to differences in solar activity in 1988 and 1996.

$\mathrm{P}$-mode frequencies from Table 1 have been inverted to investigate the structure of the solar core as reported by Fröhlich et al.(1997a) and Appourchaux et al.(1997b). From the current results alone there is no compelling evidence that the sun is substantially different from the reference model.

\section{Linewidths and Amplitudes of $\mathbf{p}$ modes}

Linewidths are determined from the same fitting procedure from both the SPM green channel and the LOI. The results are shown in Fig. 5. Note the 
TABLE 1. List of frequencies of solar $p$ modes determined from 135 days of VIRGO observations between April and August 1996.

\begin{tabular}{|c|c|c|c|c|}
\hline order & $\mathrm{l}=0$ & $\mathrm{l}=1$ & $\mathrm{l}=2$ & $\mathrm{l}=3$ \\
\hline 13 & - & - & - & $2137.79 \pm 0.07$ \\
\hline 14 & - & - & $2217.63 \pm 0.08$ & $2273.47 \pm 0.06$ \\
\hline 15 & $2228.34 \pm 0.09$ & $2292.17 \pm 0.05$ & $2352.29 \pm 0.07$ & $2407.73 \pm 0.09$ \\
\hline 16 & $2362.96 \pm 0.12$ & $2425.68 \pm 0.10$ & $2486.05 \pm 0.07$ & $2541.70 \pm 0.06$ \\
\hline 17 & $2496.14 \pm 0.09$ & $2559.29 \pm 0.07$ & $2619.69 \pm 0.08$ & $2676.23 \pm 0.05$ \\
\hline 18 & $2630.03 \pm 0.15$ & $2693.32 \pm 0.07$ & $2754.44 \pm 0.07$ & $2811.49 \pm 0.06$ \\
\hline 19 & $2764.43 \pm 0.16$ & $2828.19 \pm 0.08$ & $2889.59 \pm 0.05$ & $2946.97 \pm 0.04$ \\
\hline 20 & $2898.95 \pm 0.10$ & $2963.32 \pm 0.08$ & $3024.75 \pm 0.04$ & $3082.31 \pm 0.04$ \\
\hline 21 & $3033.75 \pm 0.09$ & $3098.16 \pm 0.11$ & $3159.83 \pm 0.07$ & $3217.74 \pm 0.05$ \\
\hline 22 & $3168.67 \pm 0.09$ & $3233.21 \pm 0.04$ & $3295.25 \pm 0.06$ & $3353.58 \pm 0.07$ \\
\hline 23 & $3303.21 \pm 0.12$ & $3368.64 \pm 0.11$ & $3430.76 \pm 0.11$ & $3489.65 \pm 0.08$ \\
\hline 24 & $3438.95 \pm 0.16$ & $3504.39 \pm 0.17$ & $3567.02 \pm 0.15$ & $3626.35 \pm 0.12$ \\
\hline 25 & $3574.62 \pm 0.29$ & $3640.35 \pm 0.24$ & $3703.41 \pm 0.22$ & $3763.01 \pm 0.18$ \\
\hline 26 & $3711.45 \pm 0.98$ & $3777.07 \pm 0.18$ & $3840.84 \pm 0.31$ & - \\
\hline 27 & $3847.28 \pm 1.57$ & - & - & - \\
\hline order & $\mathrm{l}=4$ & $\mathrm{l}=5$ & $\mathrm{l}=6$ & $\mathrm{l}=7$ \\
\hline 13 & $2188.43 \pm 0.35$ & $2235.42 \pm 0.02$ & $2280.08 \pm 0.07$ & $2322.40 \pm 0.09$ \\
\hline 14 & $2324.31 \pm 0.05$ & $2371.23 \pm 0.07$ & $2415.59 \pm 0.06$ & $2458.25 \pm 0.07$ \\
\hline 15 & $2458.53 \pm 0.05$ & $2506.00 \pm 0.06$ & $2551.12 \pm 0.05$ & $2594.27 \pm 0.06$ \\
\hline 16 & $2593.16 \pm 0.08$ & $2641.25 \pm 0.04$ & $2687.08 \pm 0.05$ & $2731.08 \pm 0.09$ \\
\hline 17 & $2728.51 \pm 0.09$ & $2777.24 \pm 0.07$ & $2823.81 \pm 0.05$ & $2868.40 \pm 0.05$ \\
\hline 18 & $2864.28 \pm 0.09$ & $2913.51 \pm 0.04$ & $2960.62 \pm 0.04$ & $3005.54 \pm 0.04$ \\
\hline 19 & $3000.13 \pm 0.05$ & $3049.84 \pm 0.05$ & $3097.09 \pm 0.05$ & $3142.77 \pm 0.04$ \\
\hline 20 & $3135.96 \pm 0.05$ & $3186.26 \pm 0.05$ & $3234.17 \pm 0.06$ & $3279.76 \pm 0.05$ \\
\hline 21 & $3271.74 \pm 0.06$ & $3322.67 \pm 0.10$ & $3370.91 \pm 0.11$ & $3417.35 \pm 0.07$ \\
\hline 22 & $3408.14 \pm 0.09$ & $3459.74 \pm 0.12$ & $3508.80 \pm 0.12$ & $3554.93 \pm 0.15$ \\
\hline 23 & $3544.67 \pm 0.10$ & $3596.35 \pm 0.17$ & $3646.05 \pm 0.14$ & - \\
\hline 24 & $3682.25 \pm 0.16$ & $3734.72 \pm 0.17$ & - & - \\
\hline
\end{tabular}

pronounced dip around the peak of the 5-minute oscillations. This dip is a resonance effect with convection which decreases the damping of the modes, and was predicted by Gough (1980; cf Balmforth, 1992). It is interesting that the IPHIR data did not show this dip, which is suggesting that the resonance effect may be decreased by increasing solar activity.

Detailed studies of the time variation of mode amplitudes for solar $\mathrm{p}$ 


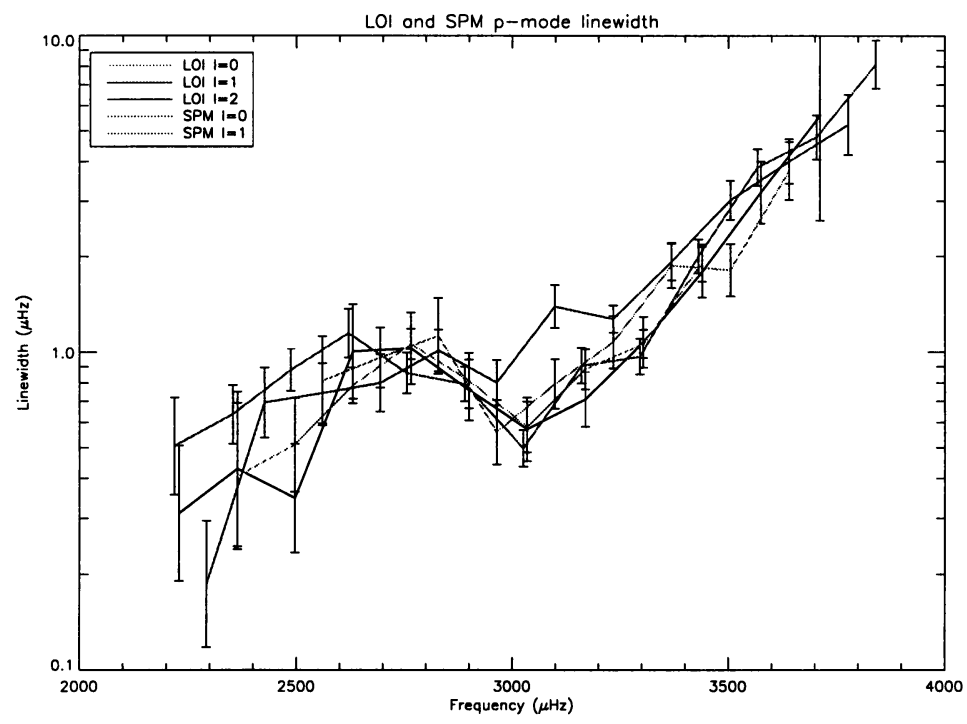

Figure 5. Linewidth determined from the SPM green channel and LOI for $l=0 \ldots 2$.

modes are essential for understanding both the excitation mechanisms and the response of the solar surface to the perturbations caused by the modes. In this context it is crucial to understand that the properties of the solar surface acts as a filter between the oscillations and the observer. These studies require continuous data sets.

Wavelet analysis is an appropriate tool for studying time-dependent phenomena. With an optimal selection of parameters this method also provides a better combined frequency and time resolution than is provided by conventional Fourier analysis. These methods have previously been successfully used on IPHIR data (Baudin et al.1994, Leifsen et al.1995). The amplitudes of the p modes from the SPM blue channel and the LOI have been studied with this method. The results from the LOI data on the $l=1$ indicate no correlation between the different rotationally split components of each radial order. This is to be expected from our view that the oscillations are excited randomly by the turbulence in the upper boundary layer of the convection zone, despite the fact that each individual excitation event applies equal impulses to eastward and westward propagating modes with like values of $l$ and $|m|$. The results with high frequency resolution for the $l=0, n=16-26$ modes from the SPM blue channel are shown in Fig. 6 . From the data we see no clear and consistent correlation of the excitation of the different modes. This too is as we expect (Chang, 1995). A prominent phenomenon is the clear variation of the central frequency position of the modes. Complicated structures with shifts and splittings of the modes are 


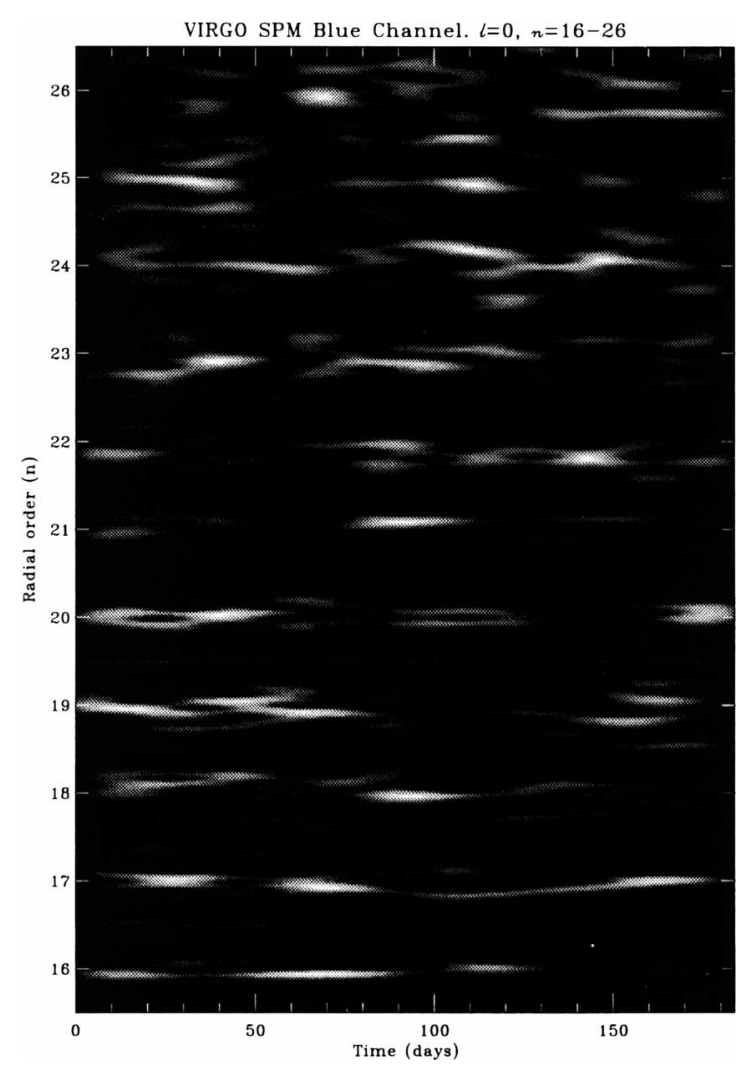

Figure 6. Wavelet analysis of $l=0, n=16 \ldots 26$ from the SPM blue channel. The time axis starts on the January 29 1996. The frequency coverage for each radial order is 4 $\mu \mathrm{Hz}$.

clearly seen. This is a result of phase wandering due to the excitation, and is manifest as a power variation in different regions of the mean Lorentzian profile of the modes. It shows the danger in fitting Lorentzians to the power spectra of modes obtained from short time strings of data. Such a fitting will give spurious frequency shifts. The required length of the time series to achieve a reasonable accuracy of the Lorentzian fitting may be so long that without appropriate care solar cycle variations could be masked.

The time dependence of the amplitude variation of the different modes has been studied by calculating the power spectra. For this, wavelet parameters giving a time resolution of about 10 hours were used. The results for $l=0$ show a dominant peak at approximately the period of the solar rotation and some minor peaks at 16.9 and 11.6 days, whereas the spectrum of the irradiance signal shows also a strong 27 day period and two other peaks at around 9 and 7 days. In the case of the irradiance they are related to 
the active region modulation as seen from time series (e.g. Fig. $1 \& 3$ ). The periodicities are also seen in the power spectra of the individual modes, but there the amplitudes of the different peaks vary strongly between the modes. The explanation of the modulation stems from the variation of the solar surface structure, i.e. with active regions, which modulate what we observe. Further observations are needed to see whether the periodicities in the amplitudes correspond to the mean solar rotation or to the rotation of the active regions.

Another interesting property of the oscillations concerns the relative amplitudes of the components of the multiplets. Although the datasets are perhaps not yet long enough to secure reliable statistics, there does appear to be a systematic variation in the power, as is illustrated in Fig. 7. Such a distribution is not inconsistent with preferential excitation of the oscillations in regions of enhanced activity. In particular, if we adopt a latitudinal variation of the excitation rate that has maxima at midlatitudes, we can account for the general trends in the observed amplitudes if the maximum excess rate of energy input into oscillations is about $50 \%$ of the uniform background. The best fit to the data of a smooth symmetric function with single maxima in each hemisphere has the maxima at about $\pm 40^{\circ}$ latitude, which is similar to though somewhat greater than the mean latitudes of the sunspot belts early in the cycle. Nevertheless, the broad agreement does suggest that magnetic activity plays a role in exciting the oscillations, in addition to modifying their frequencies (cf Kuhn and Libbrecht 1991). The difference between the locations of the maxima in the excitation function that we have deduced and the locations of the sunspots could perhaps be a result of inadequate statistics, or it could perhaps be an artifact of having misjudged the instrumental sensitivity to different modes by ignoring the magnetic perturbation to the eigenfunctions which causes the apparent decrease in power which Bogdan et al.(1993) has observed to occur in active regions. Alternatively, it could simply be that the regions of enhanced excitation extend beyond the observed regions of activity, as we were led to suspect from the variations in the radiance measurements discussed in section 3.2 .

\section{Conclusions}

All instruments within the VIRGO package work very well, and interesting new results have been gathered from our preliminary investigation of the first 4-6 months of observations. The very quiet environment of SOHO, together with the continuity of the observations, enables us to obtain time series of a quality never previously achieved. Our first results confirm broadly what has been found from previous observations: firstly, that the solar irra- 

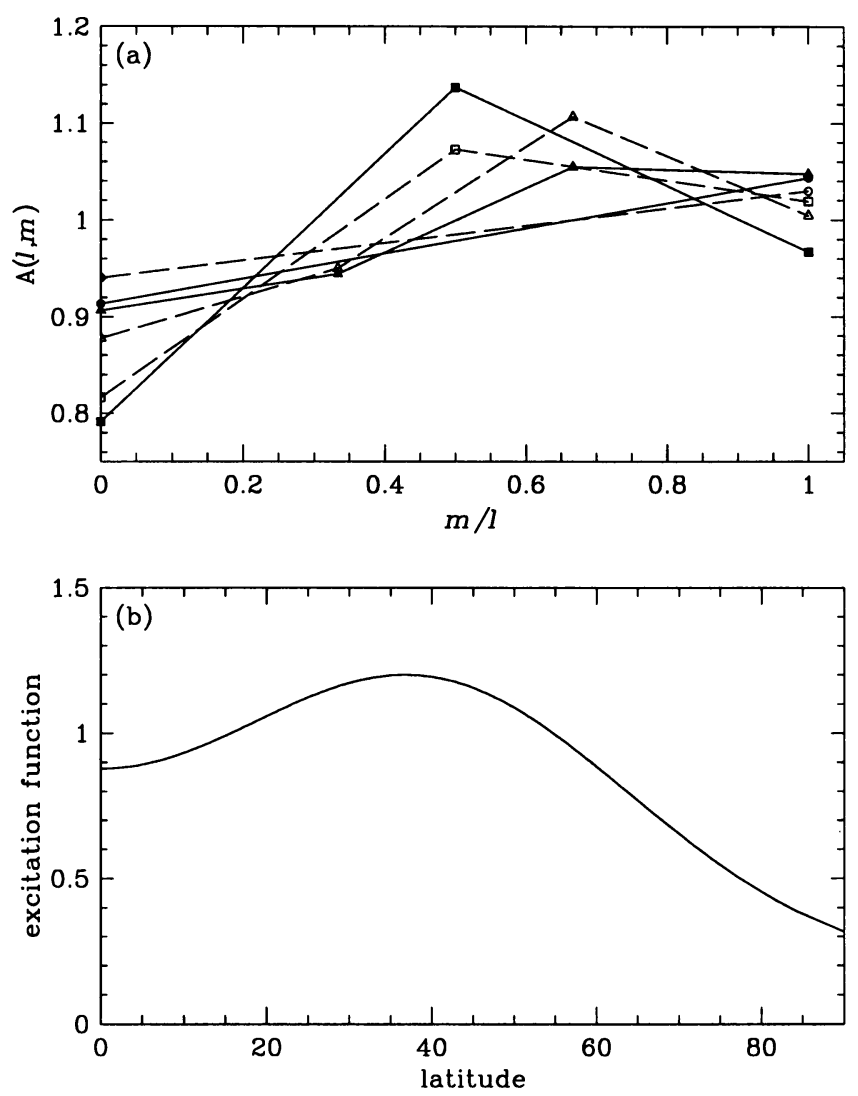

Figure 7. (a) Mean relative amplitudes of the components of dipole (circles), quadrupole (squares) and octupole (triangles) multiplets, plotted against $|m| / l$. The amplitudes of each multiplet are normalized such that the mean power per mode is unity. Filled symbols joined by continuous lines are derived from observations by LOI; the corresponding open symbols joined by dashed lines are theoretical values computed assuming the latitudinal variation of energy input illustrated in (b).

diance is modulated by the passage of sunspots across the disk, but that not all of the modulation is straightforwardly explained in terms of sunspot flux blocking and facular enhancement, and secondly, that the p-mode frequencies are, as indicated by helioseismic inversions, at present more-or-less in agreement with the latest standard solar models. However, we have in addition some new observations that provide hints that profound solar activity is more extensive than its superficial manifestation. This has been obtained partly from a comparison of coarsely resolved measures of radiance with the total irradiance. We also have evidence that magnetic activity plays a sig- 
nificant role in the dynamics of the oscillations beyond its modulation of the resonant frequencies. Pallé et al (1990) and Elsworth et al (1993) have already shown that the mean linewidths vary with the solar cycle. Comparison of our VIRGO data with IPHIR data reveals that the variation depends on frequency, in a manner that is suggestive of a modulation in the mechanism by which the oscillations interact with the turbulence in the convection zone. Moreover, by comparing the amplitudes of different components of $\mathrm{p}$-mode multiplets, each of which are influenced differently by spatial inhomogeneity, we have found evidence that activity enhances excitation. This appears to be contrary to the temporal variation reported by Pallé et al (1990) and Elsworth et al (1993), who found that on average mode amplitudes decrease as the global level of activity rises. Further analysis is required to ascertain whether this disaccord is an indication that the excitation process is substantially more complicated than one might suspect or whether the discrepancy is simply a result of the modification of the oscillation eigenfunctions in the solar atmosphere by the magnetic field. Whatever is the case, it is evident that our first results have already raised interesting issues concerning the role played by activity in the global dynamics of the sun.

\section{Acknowledgements}

VIRGO is a co-operative effort of many individual scientists and engineers at several institutes in Europe and USA. Without the continuous and concerted efforts of the team this experiment would never have reached the success it demonstrates today.

The VIRGO team has been supported by several national and international funding agencies which are gratefully acknowledged: The PMOD/ WRC by the Swiss National Science Foundation under grants 2.860-0.88, 20-28779.90, 20-33941.92, 20-40589.94 and PRODEX, the IRMB by the Fonds de la Recherche Fondamentale Collection d'initiative ministerielle and PRODEX, the SSD/ESA by their annual funds from the Science Directorate, the IAC by the CICYT through PNIE under grants ESP88-0354 and ESP90-0969, the OCA by the CNES and CNRS. Individual contributions to the project have been supported by grants from PPARC of the UK to D. O. Gough, by UCLA under a contract with NASA to J.M.Pap. Thanks are extended to the Mt.Wilson Observatory for the magnetograms retrieved from the SOHO archive.

\section{References}

Appourchaux, T., Toutain, T., Telljohan, U., Jiménez, A. and Andersen, B.N. (1995) Astr. Astrophys. 294, L13. 
Appourchaux, T., Andersen, B.N., Fröhlich, C., Jiménez, A., Telljohan, U., Wehrli, C. (1997a) Solar Phys. 170, 25

Appourchaux, T., Andersen, B.N., Berthomieu, B., Fröhlich, C., Gough, D.O., Jiménez, A., Provost, J., Sekii, T., Toutain, T., Wehrli, C. (1997b) in Schmider, F.X., Provost, J., ed(s)., Proc. IAU Symposium 181, Nice, October 1996, Kluwer Academic Publ., Dordrecht, The Netherlands, this volume

Balmforth, N.J. (1992) MNRAS 255, 603

Baudin, F., Gabriel, A. and Gibert, D. (1994) Astron.Astrophys. 285, L29

Bogdan, T.J., Brown, T.M., Lites, B.W. and Thomas, J.H. (1993) Astroph.J. 406, 723

Chang, H.-Y. (1995) Ph.D. dissertation, University of Cambridge

Crommelynck, D., Fichot, A., Domingo, V., Lee III, R. (1996) Geoph.Res.L. 23, 2293

Elsworth, Y., Howe, R., Isaak, G.R., McLeod, C.P., Miller, B.A., New, R., Speake, C.C. and Wheeler, S.J. (1993) MNRAS 265, 888

Fröhlich, C., Romero, J., Roth, H., Wehrli, C., Andersen, B.N., Appourchaux, T., Domingo, V., Telljohann, U., Berthomieu, B., Delache, P., Provost, J., Toutain, T., Crommelynck, D., Chevalier, A., Fichot, A., Däppen, W., Gough, D.O., Hoeksema, T., Jiménez, Gómez, M., Herreros, J., Roca-Cortés, T., Jones, A.R., Pap, J. and Willson, R.C. (1996) Solar Phys. 162, 101

Fröhlich, C., Andersen, B., Appourchaux, T., Berthomieu, G., Crommelynck, D.A., Domingo, V., Fichot, A., Finsterle, W., Gómez, M.F., Gough, D.O., Jiménez, A., Leifsen, T., Lombaerts, M., Pap, J.M., Provost, J., Roca Cortés, T., Romero, J., Roth, H., Sekii, T., Telljohann, U., Toutain, T., Wehrli, C. (1997a) Solar Phys. 170, 1

Fröhlich, C., Crommelynck, D., Chevalier, A., Fichot, A., Finsterle, W., Jiménez, A., Romero, J., Roth, H., Wehrli, C. (1997b) Solar Phys. 173, submitted

Gough, D.O. (1980) in Hill, H.A., Dziembowski, W.A., ed(s)., Nonradial and Nonlinear Stellar Pulsation, Lecture Notes in Physics 125, Springer-Verlag, Berlin, 273

Harvey, J. (1985) E.Rolfe \& B.Battrick Future missions in solar, heliospheric \& space plasma physics, SP 235: ESA Publication Division, Noorddwijk 199

Kuhn, J.R. (1996) in T. Roca-Cortes \& F. Sánchez, ed(s)., The structure of the Sun, Cambridge University Press, 231

Kuhn, J.R. and Libbrecht, K.G. (1991) ApJ. 381, L35

Leifsen, T., Hanssen, A., Andersen, B. N. and Toutain, T. (1995) A.S.P. Conf. Ser. 76, 520

Neckel, H., Labs, D. (1984) Sol.Phys. 90, 205

Solar-Gephysical Data (1996) Solar-Gephysical Data comprehensive reports Number 624, Part II, 24

Pallé, P.L., Régulo, C. and Roca Cortés, T. (1990) in Y. Osaki \& H. Shibahashi, ed(s)., Progress of Seismology of the Sun and Stars, Lecture Notes in Physics 367, Springer Verlag, Berlin, 189

Toutain, Th. and Fröhlich, C. (1992) Astr. Astrophys. 257, 287.

Willson, R.C., Gulkis,S.,Janssen,M., Hudson, H.S., Chapman.G.A. (1981) Science 211, 700 